\title{
Characterization of EBL2 EUV exposure facility
}

Edwin te Sligte, Michel van Putten, Freek T. Molkenboer, Peter van der Walle, Pim M. Muilwijk, et al.

Edwin te Sligte, Michel van Putten, Freek T. Molkenboer, Peter van der Walle, Pim M. Muilwijk, Norbert B. Koster, Jeroen Westerhout, Peter J. Kerkhof, Bastiaan W. Oostdijck, Wouter Mulckhuyse, Alex F. Deutz, "Characterization of EBL2 EUV exposure facility," Proc. SPIE 10450, International Conference on Extreme Ultraviolet Lithography 2017, 1045027 (16 October 2017); doi: 10.1117/12.2280356

SPIE Event: SPIE Photomask Technology and EUV Lithography, 2017, Monterey, California, United States 


\title{
Characterization of EBL2 EUV exposure facility
}

\author{
Edwin te Sligte*, Michel van Putten, Freek T. Molkenboer, Peter van der Walle, Pim M. Muilwijk, \\ Norbert B. Koster, Jeroen Westerhout, Peter J. Kerkhof, Bastiaan W. Oostdijck, Wouter \\ Mulckhuyse, Alex F. Deutz \\ TNO, Stieltjesweg 1, 2628 CK Delft, The Netherlands
}

\begin{abstract}
TNO has built EBL2; a facility for EUV exposure testing and surface analysis. EBL2 is capable of testing EUV optics, EUV photomasks, pellicles, and other components under controlled conditions, relevant to EUV scanner and source operation at all foreseen source power nodes. The system consists of an EUV beam line coupled to an X-ray Photoelectron Spectroscopy system by an automated sample handler. The current contribution reports on the results of the qualification testing of the EUV beam line. Topics investigated include handling and position control, thermal management, a relevant gas environment, EUV irradiation and metrology, and first EUV exposures.
\end{abstract}

Keywords: EUV exposure, mask, pellicle, XPS analysis, metrology, handling, contamination control

\section{INTRODUCTION}

EUV is approaching its insertion point for high volume manufacturing as EUV sources and EUV lithography tools become increasingly mature products ${ }^{[1]}$. At the same time, there is a continued push for EUV source powers beyond $250 \mathrm{~W}^{[2]}$, which means optics, reticles and pellicles will have to withstand increasing EUV intensities for increasing amounts of time. This development leads to a growing need for EUV exposure testing capability.

TNO has built the EBL2 facility for EUV exposure testing and surface analysis to meet this demand. EBL2 is capable of testing EUV optics, EUV photomasks, pellicles, and other components under controlled conditions, that are relevant to EUV scanner and source operation at all foreseen source power nodes. This enables life time testing of EUV optics, reticles and pellicles under conditions which are not yet available to industry. This predictive testing will help the industry to prepare for high volume production.

\section{SYSTEM OVERVIEW}

This section aims to give a brief overview of the EBL2 system; a full description can be found in refs. [3-4]. The layout of the system is shown in Figure 1, along with a photograph of the EBL2 clean room. EBL2 consists of an EUV beam line coupled to an X-ray Photoelectron Spectroscopy (XPS) system by an automated sample handler.

The EBL2 beam line is the EUV exposure part of the facility. It consists of an EUV source, a Collector Module, and an Exposure Chamber. The Ushio EUV source is described in Ref [5], and generates EUV from a Sn Laser assisted Discharge Plasma. The EUV source is mounted on a track, which allows it to be separated from the Collector Module to facilitate maintenance. The Collector Module contains two elliptical mirrors that project EUV into the Exposure Chamber. It also provides the option to load Spectral Purity Filters into the EUV beam. A vacuum separation between the Source and Exposure Chamber gas environments is present at the intermediate focus of the two mirrors. The EUV beam focus can be adjusted relative to the sample by moving the source and collector module along a track. The exposure chamber contains a sample chuck positioned by a hexapod mounted outside the vacuum system. A Sample Rotation Unit (SRU) is present to rotate the mask or sample holder, and a gas manifold creates the desired gas environment. An in situ imaging ellipsometer keeps track of the sample condition during EUV exposures.

The Kratos XPS system is used for surface analysis of the mask or sample under study ${ }^{[6]}$. This XPS can be reached without breaking vacuum from the beam line by an automated Vacuum Handler. The Vacuum Handler can also move the mask or sample to a Parking and Cleaning station, where it can be cleaned using atomic hydrogen. The sample can

\footnotetext{
International Conference on Extreme Ultraviolet Lithography 2017, edited by Paolo A. Gargini,

Patrick P. Naulleau, Kurt G. Ronse, Toshiro Itani, Proc. of SPIE Vol. 10450, 1045027

(c) 2017 SPIE $\cdot$ CCC code: $0277-786 X / 17 / \$ 18 \cdot$ doi: $10.1117 / 12.2280356$
} 
also be stored in vacuum in a Storage chamber. Samples enter and leave the vacuum system of EBL2 through a load lock. An Atmospheric Handler unloads the samples or masks from an EUV Dual Pod, rotates and flips them as needed, and transfers them to the load lock in the appropriate orientation.

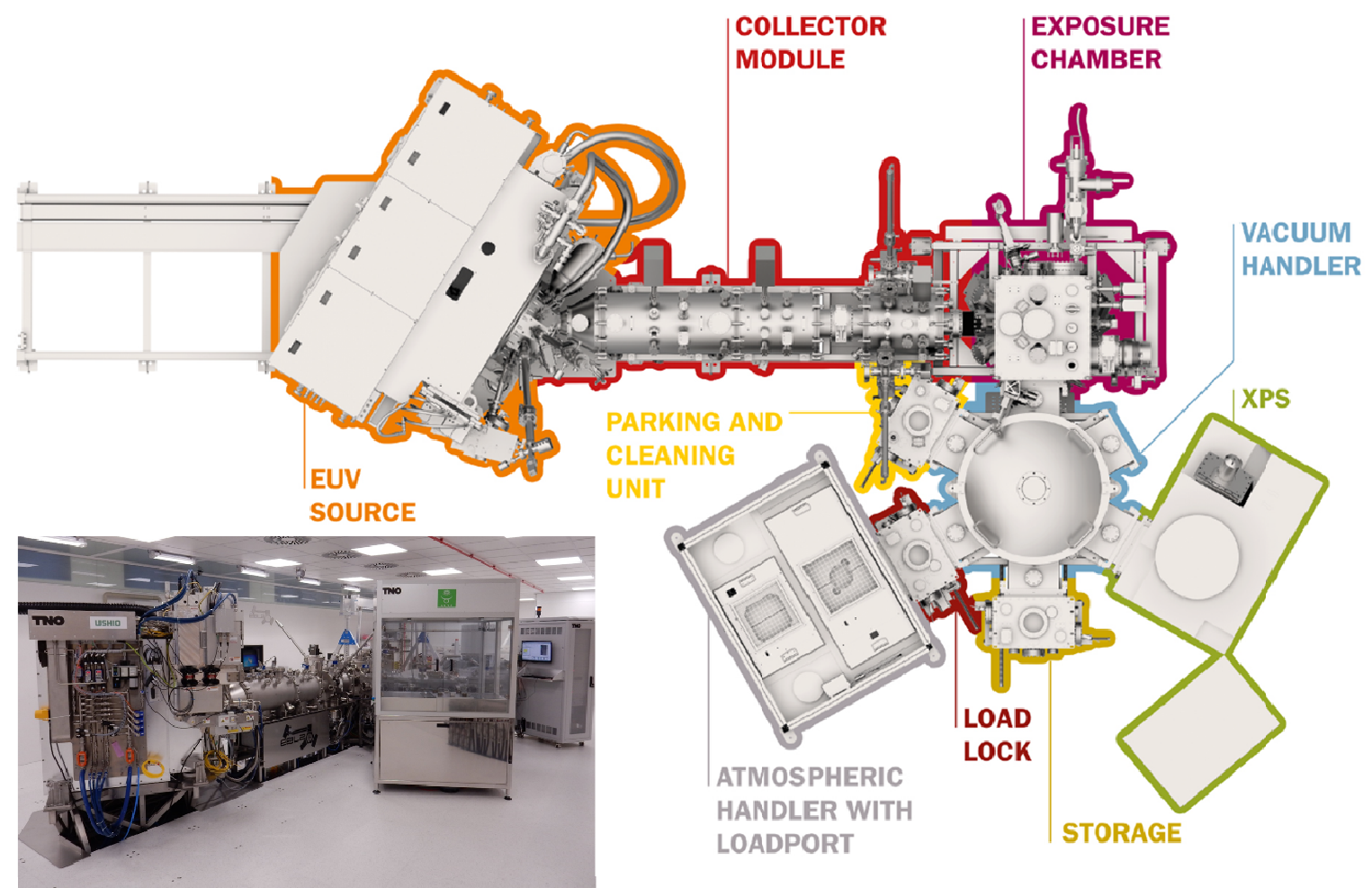

Figure 1: Overview of EBL2 facility. Inset: photograph of the EBL2 clean room.

The full system accepts standard EUV photomasks, with or without pellicles. Smaller samples can be handled using sample holders with generic interfaces to the EBL2 system. Both handlers and other modules maintain reticle backside cleanliness to enable loading of used masks into an NXE machine. This enables wafer printing evaluation of masks that have been exposed on EBL2.

The system was realized in close collaboration with our technology partners Ushio Inc for the Sn fueled EUV source and ASYS Automatic Systems GmbH \& Co. KG for both the vacuum and atmospheric handler. The beam line reached its First Light milestone in late $2016^{[7]}$, and has since been subjected to verification and validation testing. The remainder of this paper provides results of this testing, and shows the first EUV exposures on EBL2.

\section{SAMPLE LOADING AND POSITIONING}

The first step in an EUV exposure is loading the sample onto the chuck in the Exposure Chamber. As described in Ref. [3], the mask is handled quality side up by a vacuum robot, but is exposed in vertical orientation. The automated procedure for loading photomasks into the Beam Line is illustrated in Figure 2.

a. The Vacuum Handler robot extends into the Exposure Chamber of EBL2, and positions the mask between the arms of the Sample Rotation Unit (SRU).

b. The SRU arms close, grabbing the mask.

c. The Vacuum Handler robot lowers the base plate, and retracts it from the Exposure Chamber.

d. The SRU rotates the mask so that the quality side faces the EUV beam path.

e. The chuck moves in from behind and positions itself to clamp the mask.

f. The chuck clamps the mask, after which the SRU arms release.

After this procedure, the chuck is free to move the mask to the desired exposure location. 

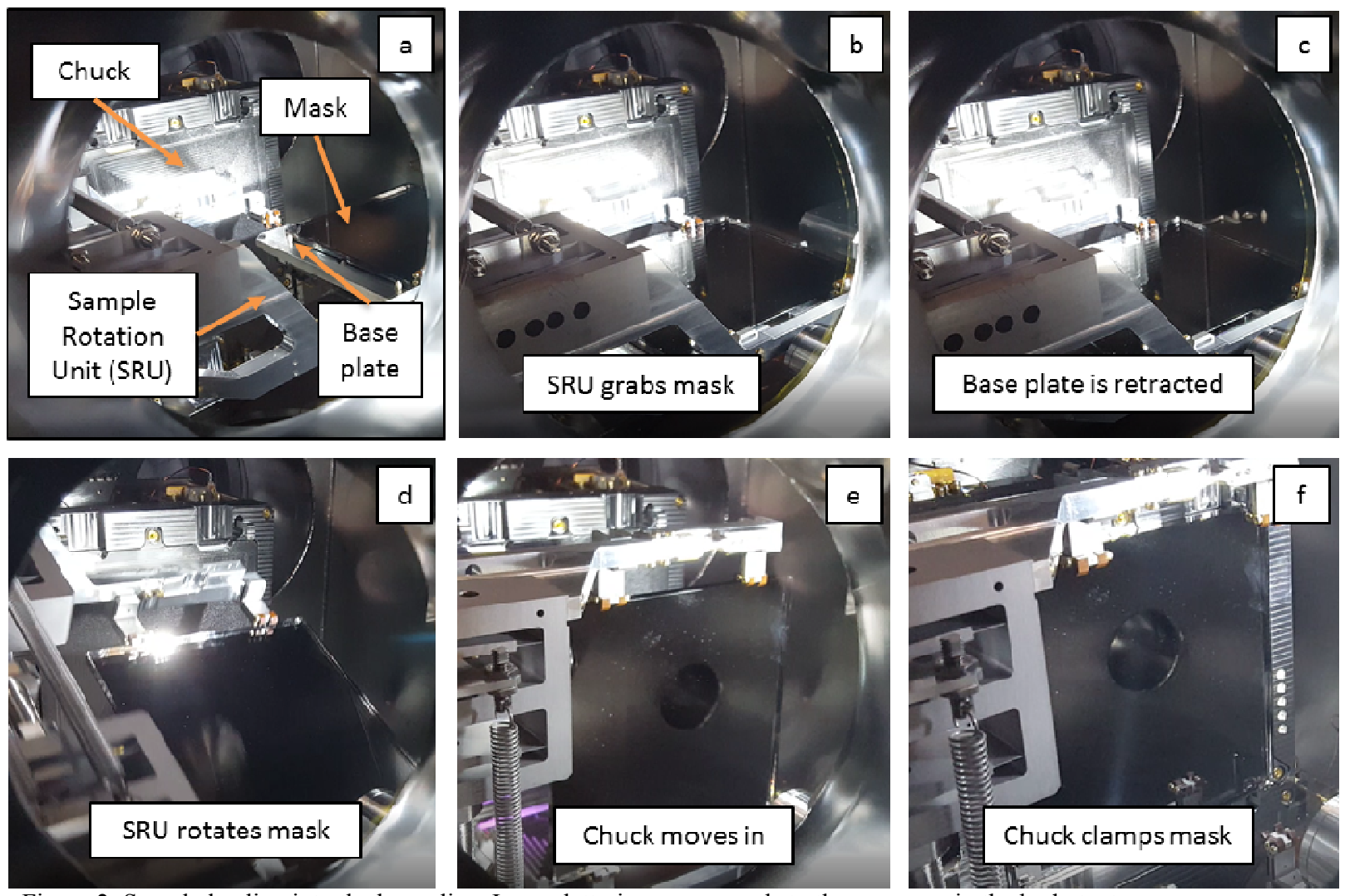

Figure 2: Sample loading into the beam line. Image lettering corresponds to the sequence in the body text.

Moving the mask to the desired exposure position requires a measurement of the position of the mask relative to the EUV beam. This measurement is performed using a scintillator crystal which is rigidly mounted on the chuck. EBL2's in-situ imaging ellipsometer and scintillator camera are used in a correlated measurement of alignment marks on the scintillator, as illustrated in Figure 3. The EUV beam impinges on the scintillator from the right. The scintillator is coated to reject any visible light coming from the EUV source. A camera looking at the back of the scintillator captures the EUV spot profile. A sample spot profile image is shown on the left. The position of the EUV spot relative to the crystal can be determined using the cross-hair alignment marks that are embossed on the scintillator coating. At the same time, the imaging ellipsometer can simultaneously assess the position of the cross-hairs in its field of view from the front side. As the EUV scintillator camera and imaging ellipsometer both detect the same crosshairs, the position of the EUV beam relative to the stage is known.

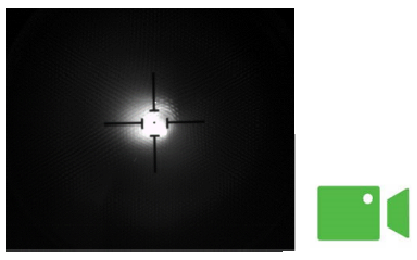

Scintillator camera
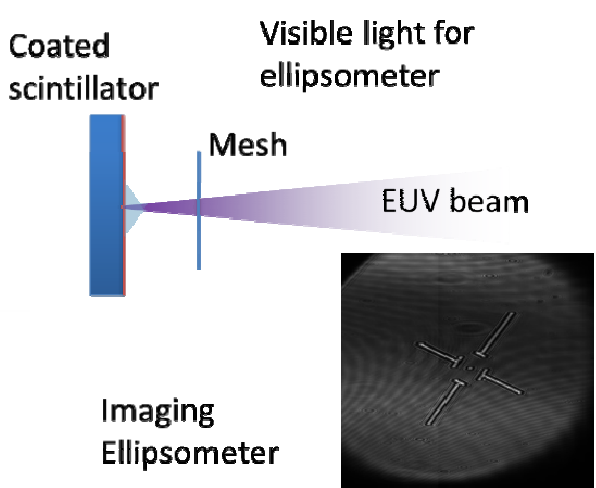

Figure 3: EUV beam to sample stage alignment scheme. The same crosshairs are inspected from the front by visible light, and from behind by EUV scintillation. 
Once the EUV beam position is correlated to the chuck coordinates, the imaging ellipsometer can resolve alignment marks on the mask, and the mask can be moved to correlate with the EUV beam. The distance between the cross-hairs of the scintillator is $2 \mathrm{~mm}$; the imaging resolution of both the ellipsometer and scintillator camera is approximately $50 \mu \mathrm{m}$, enabling a mask positioning accuracy better than $100 \mu \mathrm{m}$.

\section{GAS AND THERMAL MANAGEMENT}

The gas environment during an exposure is critical to accurately simulate the operational environment. This requires a low base pressure, pure operating gases, and the ability to add contaminants in a controlled way. In operation, the background pressure without added gases is less than $5 \cdot 10^{-7}$ mbar; this pressure is dominated by the Ar purge gas used in the EUV source. The design target for the operational base pressure of $1 \cdot 10^{-6}$ mbar was met. The available operating gases are $\mathrm{H}_{2}, \mathrm{He}$, and Ar; $\mathrm{N}_{2}, \mathrm{O}_{2}, \mathrm{XCDA}$, and hydrocarbons are available as additives. Pressure control for all these gases meets the design targets.

As EUV requires vacuum, active cooling is critical to control sample temperature. EBL2 uses backfill gas to establish thermal contact between the cooled chuck and the mask. The chuck can be cooled to $-20^{\circ} \mathrm{C}$ with temperature control using a resistive heater and a PID circuit. The performance of the chuck thermal control is illustrated in Figure 4. The chuck temperature is set to $-20^{\circ} \mathrm{C}$ during an exposure. It remains stable within $0.5^{\circ} \mathrm{C}$ of its set point when the EUV irradiation is switched off, demonstrating that the temperature control works as intended.

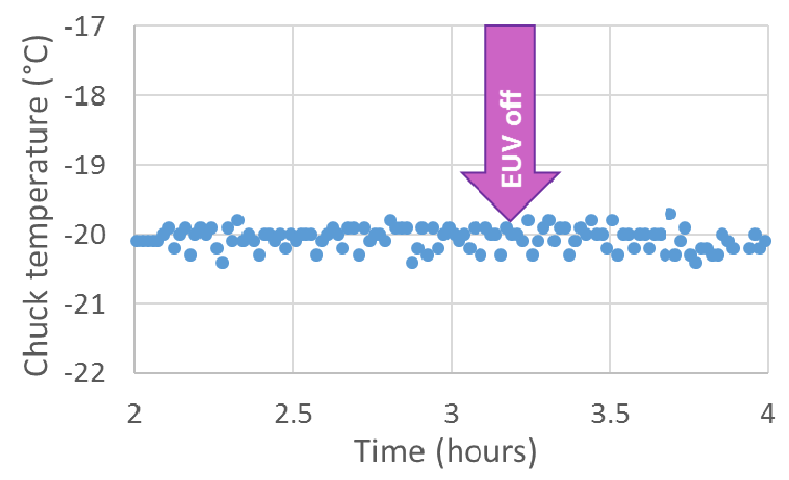

Figure 4: Thermal control performance

\section{EUV METROLOGY}

Measurement of the EUV power and intensity is critical to the EUV irradiation functionality of EBL2. A scintillator is present to measure EUV spot profiles, as discussed above. The EUV intensity can be measured using two photodiodes mounted on the stage. Both diodes were calibrated at PTB, and are mounted behind a pinhole to prevent overloading the photodiodes. The areas of the pinholes were measured.

An intensity measurement is carried out by moving one of the photodiodes to the location of interest, or making a line scan through the EUV beam. The photocurrent of each diode is monitored, and for each EUV pulse, the total charge generated is calculated. Using the calibration value, the total EUV pulse energy is derived, which is converted to power by multiplication by the repetition rate. Finally, division by the known surface area of the pinhole results in an intensity value in $\mathrm{W} / \mathrm{mm}^{2}$.

In Figure 5, two sample EUV intensity scans are shown. The scans were taken before and after a three hour EUV exposure, with no alignment changes in between. The differences are negligible, indicating that the system alignment and source power output are stable. Some shot noise can be seen in the curves, as each data point was acquired from a single EUV pulse. 


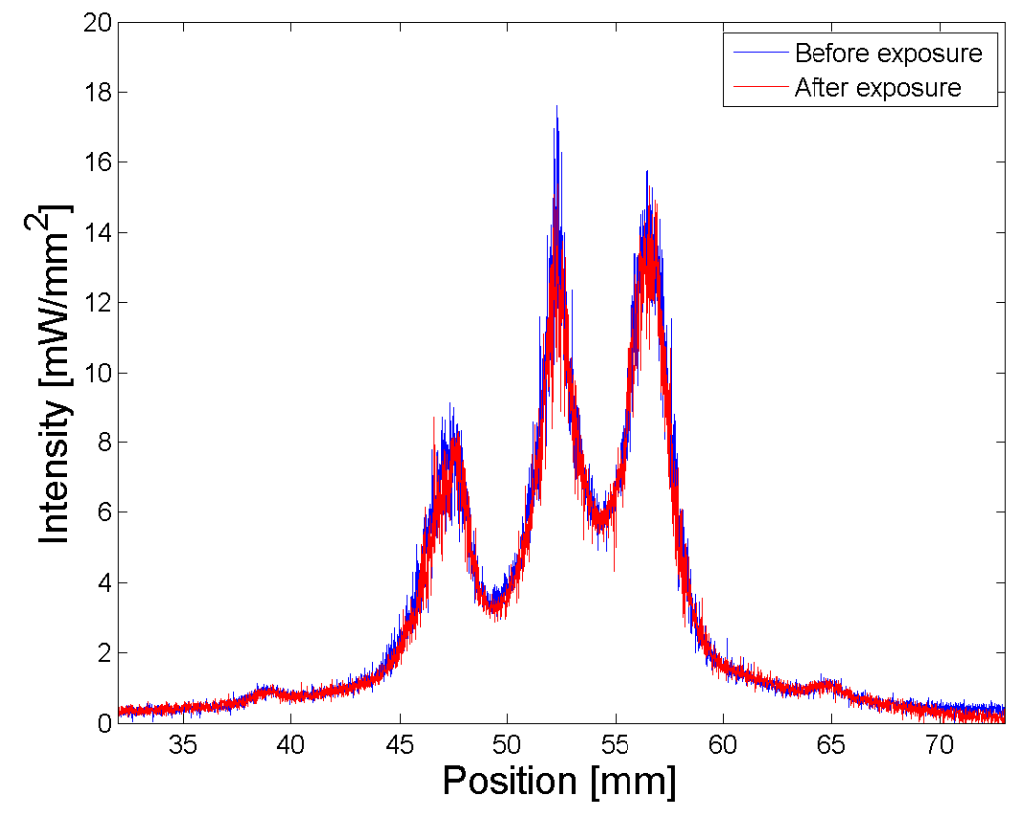

Figure 5: Sample EUV intensity measurements by scanning the photodiode through the EUV beam

\section{CURRENT STATUS AND OUTLOOK}

Currently, all EBL2 subsystems have been tested, and the system is running trial exposures. The beam line alignment is being optimized, and operational protocols are being generated. Two trial exposures are illustrated in Figure 6 below. The image on the left shows a trial exposure on an EUV mask without operating gas. As the visible light that travels from the source with the EUV beam is diffracted by the patterned areas of the mask, these areas are seen to light up in a ring-shaped profile. The image on the right shows an exposure using a sample holder that holds several 1" diameter samples. One of these samples is irradiated in a hydrogen atmosphere. As the EUV light ionizes the hydrogen gas, a plasma forms in the beam path. The radiation emitted by this plasma allows the focus of the EUV beam to be seen in front of the sample. This effect is notably absent in the left image in Figure 6.
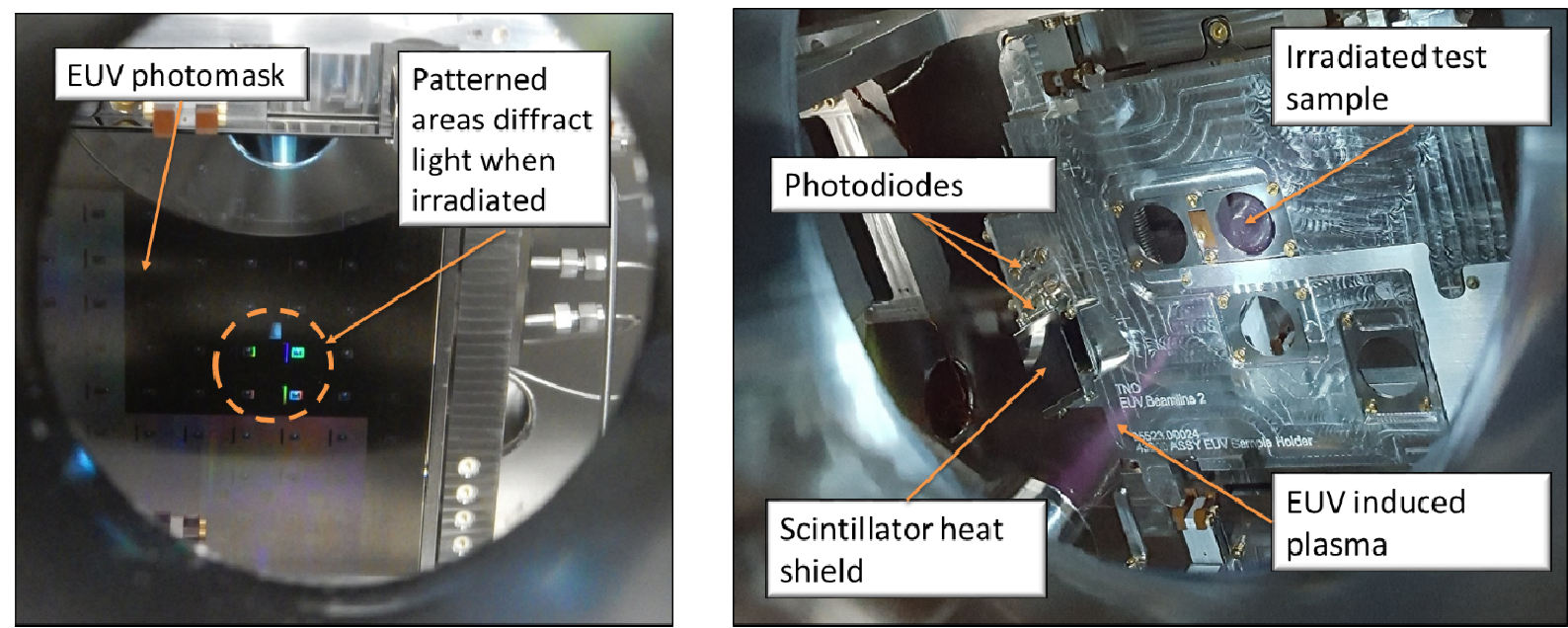

Figure 6: Left: mask exposure in EBL2, no process gas. Right: test sample exposure in $\mathrm{H}_{2}$. 
EBL2 is operational as a flexible EUV test tool for masks, pellicles, and other samples. In the near future, the remaining test program will be completed, and the system will be used to develop a mask lifetime test protocol. The system is now available for all users.

\section{ACKNOWLEDGEMENTS}

TNO would like thank its technology partners Ushio, Inc. and ASYS Automatic Systems GmbH \& Co. KG for the open and constructive collaboration in designing EBL2, as well as the Advanced Mask Technology Center GmbH \& Co. KG for providing the test mask. TNO would also like to thank the potential users of EBL2 in helping with defining the right requirements and needs for EBL2. EBL2 is a TNO initiative and realization was made possible with funding from the European Union (SENATE and E450LMDAP project), Dutch ministry of Economic Affairs (NanoLabNL and Toekomstfonds).

\section{REFERENCES}

[1] Britt Turkot, Steven L. Carson, Anna Lio, Ted Liang, Mark Phillips, Brian McCool, Eric Stenehjem, Tim Crimmins, Guojing Zhang, Sam Sivakumar, "EUV progress toward HVM readiness", Proc. SPIE 9776, Extreme Ultraviolet (EUV) Lithography VII, 977602 (March 18, 2016); doi: 10.1117/12.2225014

[2] Alexander A. Schafgans, Daniel J. Brown, Igor V. Fomenkov, Yezheng Tao, Michael Purvis, Slava I. Rokitski, Georgiy O. Vaschenko, Robert J. Rafac, David C. Brandt, "Scaling LPP EUV sources to meet high volume manufacturing requirements (Conference Presentation)", Proc. SPIE 10143, Extreme Ultraviolet (EUV) Lithography VIII, 101431I (2017/05/05); doi: 10.1117/12.2258628

[3] Edwin te Sligte, Norbert Koster, Freek Molkenboer, Alex Deutz, "EBL2, a flexible, controlled EUV exposure and surface analysis facility", Photomask Japan (2016), DOI: 10.1117/12.2240302

[4] Edwin te Sligte, Norbert Koster, Freek Molkenboer, Peter van der Walle, Pim Muilwijk, Wouter Mulckhuyse, Bastiaan Oostdijck, Christiaan Hollemans, Björn Nijland, Peter Kerkhof, Michel van Putten, André Hoogstrate, Alex Deutz, "EBL2: high power EUV exposure facility”, Photomask Technology (2016), Proc. of SPIE Vol. 9985, 998520-1, doi: 10.1117/12.2240921

[5] Yusuke Teramoto, Bárbara Santos, Guido Mertens, Ralf Kops, Margarete Kops, Alexander von Wezyk, Hironobu Yabuta, Akihisa Nagano, Takahiro Shirai, Noritaka Ashizawa, Kiyotada Nakamura, Kunihiko Kasama, "Highradiance LDP source for mask-inspection application," in Extreme Ultraviolet (EUV), Lithography VI, Proc. of SPIE Vol. 9422 (SPIE, San Jose, CA, March 2015), pp. 94220F-1-9

[6] Norbert Koster, Edwin te Sligte, Alex Deutz, Freek Molkenboer, Pim Muilwijk, Peter van der Walle, Wouter Mulckhuyse, Bjorn Nijland, Peter Kerkhof, Michel van Putten, "First light and results on EBL2", Proc. SPIE 10454, Photomask Japan 2017: XXIV Symposium on Photomask and Next-Generation Lithography Mask Technology, 104540 O (2017/07/13); doi: 10.1117/12.2279025

[7] Norbert Koster, Edwin te Sligte, Freek Molkenboer, Alex Deutz, Peter van der Walle, Pim Muilwijk, Wouter Mulckhuyse, Bastiaan Oostdijck, Christiaan Hollemans, Björn Nijland, Peter Kerkhof, Michel van Putten, Jeroen Westerhout, "First light at EBL2", Proc. SPIE 10143, Extreme Ultraviolet (EUV) Lithography VIII, 101431N (2017/03/24); doi: 10.1117/12.2257997 